\title{
Towards a predictive model for polycyclic aromatic hydrocarbon dimerization propensity
}

\author{
Jeffrey S. Lowe ${ }^{\mathrm{a}}$, Jason Y.W. Lai ${ }^{\mathrm{b}}$, Paolo Elvati ${ }^{\mathrm{b}}$, Angela Violi ${ }^{\mathrm{c}, *}$ \\ ${ }^{a}$ Department of Chemical Engineering, University of Michigan, Ann Arbor, MI 48109-2136, \\ $U S A$ \\ ${ }^{b}$ Department of Mechanical Engineering, University of Michigan, Ann Arbor, MI \\ 48109-2125, USA \\ ${ }^{c}$ Departments of Mechanical Engineering, Chemical Engineering, Biomedical Engineering, \\ Macromolecular Science and Engineering, and Applied Physics, University of Michigan, \\ Ann Arbor, MI, 48109, USA
}

Colloquium: Soot, PAH, \& Other Large Molecules

Total Length of Paper: 5758 Words, Method 2

Main Text $=3500$

References $=900$

1 Table $=140$

4 Figures $(450+350+154+264)=1218$

${ }^{*}$ Corresponding author.

Tel: +1(734)615-6448; Fax +1(734)647-9379

E-mail Address: avioli@umich.edu

Postal Address: 2250 G.G. Brown Laboratory, 2350 Hayward Street, Ann Arbor, MI, 481092125, USA

Preprint submitted to Proceedings of the Combustion Institute

May 27, 2014

(C) 2014. This manuscript version is made available under the Elsevier user license http://www.elsevier.com/open-access/userlicense/1.0/ 


\begin{abstract}
Soot particles are a significant pollutant formed as the result of incomplete combustion. Particle nucleation significantly impacts the formation and morphology of soot particles, yet remains a key knowledge gap. To elucidate the process of nucleation, we have investigated the thermodynamic stability of dimers of polycyclic aromatic hydrocarbons (PAHs), towards developing a more comprehensive model for PAH clustering behavior. Using a computational methodology based on molecular dynamics and well-tempered Metadynamics, we quantified the impact of morphological parameters on homo-molecular dimerization, as well as the relative size of monomers on the stability of hetero-molecular dimers. The results illustrated the substantial impact of PAH mass and geometry on the stability of homo-molecular and hetero-molecular dimers at flame temperatures. In particular, dimer stability was found to depend most strongly on monomer mass, followed by solvent-accessible surface area. Additionally, hetero-molecular dimer stability was found to be largely determined by the size of the smallest monomer. Identifying relationships between PAH morphology and thermodynamic stability is a significant step towards a more comprehensive understanding of the physical interactions between PAHs. Altogether, this work presents a framework for elucidating the clustering behavior of arbitrary PAHs and will greatly impact understanding and modeling of particle nucleation and growth.
\end{abstract}

\title{
Keywords
}

Soot, Polycyclic Aromatic Hydrocarbons, Dimerization, Nucleation, Clustering 


\section{Introduction}

Particle inception, or nucleation, which delineates the transition from the gas to solid phase, is a key knowledge gap in soot formation. There is a substantial body of work, reviewed previously [1-9], that has investigated soot formation in combustion. This work shows that inception is a complex process involving both chemical and physical phenomena [3] and has a significant impact on the growth of combustion-emitted particles, especially their size distribution [10] and morphology. Importantly, although nucleation is expected to involve polycyclic aromatic hydrocarbons (PAHs), the exact mechanisms involved remain poorly understood. The most prevalent proposed mechanism for nucleation is the physical agglomeration of moderately sized PAHs into clusters [1]. Specifically, the irreversible dimerization of pyrene $\left(\mathrm{C}_{16} \mathrm{H}_{10}\right)$ [11] is the most widely used definition of inception in numerical models [2, 3]. However, more recent work has argued against the plausibility of pyrene dimerization as a key nucleation step [1, 12-16]. For example, experimental observation of pyrene dimerization kinetics showed that at high temperatures, equilibrium favors pyrene dissociation [13].

Improvements in particle inception models have been proposed, but these models remain relatively simple. For example, the Polycyclic Aromatic Hydrocarbon - Primary Particle (PAH-PP) model $[17,18]$ describes the formation, growth, and oxidation of soot particles in premixed laminar flames, but is based on empirical parameters that must be tuned to fit with experimental data. The PAH-PP model defines an incipient particle nucleus as a dimer of two PAHs. Dimerization occurs upon collision of planar PAHs with the empirical sticking probability that depends on the surface area and mass of the colliding particles [19]. A model suggested by D'Anna et al. [20] aims to relate dimerization efficiency with dispersion forces and uses a sticking coefficient that is dependent on the interaction potential between colliding particles. The interaction potential is estimated through molecular mass and the Hamaker constant. The latter value was determined for PAHs by analogy with macroscopic material constants 
such as graphite.

Given the uncertainty surrounding inception, a more accurate model of PAH agglomeration will greatly benefit soot particle modeling efforts. Previously, we demonstrated a computational methodology for quantifying the stability of PAH clusters [15] in terms of thermodynamic free energy. The current work discusses development towards a more comprehensive model for PAH clustering behavior through quantification of the impact of morphology. Specifically, we investigated the impacts of mass; the presence of five- and six-membered rings; the morphology of PAHs; and the relative size of monomers of hetero-molecular dimer pairs. Significant correlations were observed, highlighting the substantial impact of mass and geometry on the physical interactions of homo-molecular and hetero-molecular monomer pairs. Moreover, our model adds insight on the entropic contribution to dimer stabilization, which plays an important role at flame temperatures in determining the resistance to binding between two particles. This represents a significant step towards a more comprehensive predictive capability for physical interactions between PAHs and will greatly impact understanding of particle nucleation, and growth.

\section{Methodology}

In the current work, we employed a previously validated methodology, described in detail elsewhere [15], for determining the thermodynamic stability of PAH clusters using atomistic simulation. This methodology utilizes molecular dynamics (MD) coupled with the well-tempered Metadynamics algorithm [21] to reconstruct the Helmholtz free energy surfaces (FESs) of the dimerization of PAH molecules. We computed the free energy surface for each monomer pair as follows. First, we prepared a system, consisting of two molecules, by performing a relaxation run of $1 \mathrm{~ns}$ in the canonical ensemble in a periodic box with a side length of $10 \mathrm{~nm}$. Second, we ran a $350 \mathrm{~ns}$ free energy reconstruction simulation from the final configuration of the relaxation run at the target temperature $(500,1000$, or $1500 \mathrm{~K})$. We used the distance between the 
center of mass of each monomer as the collective variable for the free energy reconstructions. We employed the parameters of Herdman and Miller [22] for the non-bonded potential between aromatic hydrocarbons, and the Optimized Potentials for Liquid Simulations - All Atom (OPLS-AA) [23] force field for the remaining interaction parameters. The Herdman and Miller parameters have been optimized for a large system of homo- and hetero-molecular clusters of PAHs over a broad range of monomer masses and the OPLS-AA force field was adapted for liquid- and gas-phase species. The reader is directed to our previous study for a more detailed analysis of our choice of potentials [15]. For larger molecules, a particle-particle particle-mesh solver was employed to account for long-range electrostatic interactions. The remaining parameters used in our simulations are detailed in Table 1 . Note that the bias factor is highly dependent on the type of molecule studied; therefore, the general interval is reported. For the MD simulations, we used the LAMMPS code [24] compiled with the PLUMED 1.3.0 plugin [25]. We analyzed configurational parameters by visualizing molecules with VMD 1.9.1 [26].

\section{Results and Discussion}

Figure 1 shows the pool of investigated molecules, some of which possess established names. The chosen molecules include pericondensed six-membered ring aromatics up to a size of 96 carbons, most of which are the stabilomers defined by Stein and Fahr [27] that are commonly proposed as significant PAHs in combustion. The second set of studied molecules was chosen based on a recent investigation of PAH growth, using a newly developed code, the Stochastic Nanoparticle Simulator (SNAPS), and a PAH chemical growth mechanism [28]. SNAPS, a substantial development of AMPI [29], utilizes an algorithm based on kinetic Monte Carlo to explore molecules' structures that can be produced from a given chemical reaction mechanism. The molecules chosen for this work were frequently observed in simulations of a premixed laminar flame. To distinguish these molecules from the first group they are labeled as M1-M7. Note 
that this second set of molecules contains compounds with both five- and sixmembered rings. We quantified thermodynamic stability for a given dimer using the free energy difference between the dimer and monomer states, referred to as dimer stability (DS). The dimer state corresponds to a free energy minimum in instances where a well exists, typically around $0.4 \mathrm{~nm}$. The monomer state corresponds to positions on the free energy curve where molecules are far apart and interaction between them is minimal, typically for distances greater than about $1.7 \mathrm{~nm}$.

To begin our analysis we evaluated the impact of molecule size (mass) on DS. This was chosen as our starting point because many studies suggest that mass is an important factor in determining the dimerization stability $[13,15,16]$. Figure $2 \mathrm{a}$ shows the dependency on the aromatic carbon number of the free energy of dimerization for the homo-molecular dimerization process of the purely aromatic molecules at different temperatures. The plot demonstrates that DS is strongly dependent on carbon number and that DS approaches an asymptotic value for large molecules. This implies that homo-molecular dimers above a certain size will have roughly equivalent stabilities. The trend we observe for free energy is very similar to the results Herdman and Miller observed for binding energy [22]. The difference lies in the inclusion of the entropic terms in the current study, which have a strong temperature dependence. The temperature effect is nearly linear, causing a destabilization of the dimer of about $1 \mathrm{~kJ} / \mathrm{mol}$ per carbon atom every $500 \mathrm{~K}$. This loss in DS is due to the entropic contribution, causing a favorability of the monomer states. We expect the observed trend since entropy has the effect of lowering the free energy curve of individual dimers. The simple temperature dependence allows us to extrapolate the DS to higher or lower temperatures by linear fitting.

The results in Figure 2a were then used as a first approach towards the development of predictive trends for quantifying DS. Following the functional form Herdman and Miller used for binding energy [22], the data in Figure 2a were fit to the relationship $F E / N=a_{0}-a_{1}\left(N-a_{2}\right)^{-1}$ where FE is the free energy dimer stability per carbon atom, $\mathrm{N}$ is the number of carbon atoms in a 
monomer, and $a_{0}, a_{1}$, and $a_{2}$ are the fitted constants(see Tables S1-S3 in the Supplementary Material).

Next we analyzed DS with regard to molecular morphology. The results of our previous study hinted at the importance of molecular structure in affecting the stability of dimers [15]. Here we account for particle morphology by measuring the solvent-accessible surface area (SASA) of a molecule. The SASA is equivalent to the surface area that is accessible by a solvent if a molecule were immersed in a fluid. Algorithms for computing SASA calculate the area accessible by a probe that is rolled over the surface of a molecule with its atoms represented by van der Waals radii. It is expected that structures with a greater SASA will have a lower dimerization propensity because they will have a lessdense arrangement of carbon atoms and therefore weaker interactions. The relationship between DS and SASA is displayed in Figure $2 \mathrm{~b}$ at $1000 \mathrm{~K}$. A similar result was achieved at 500 and $1500 \mathrm{~K}$ (Tables S4 and S5). These data show a linear correlation between DS and SASA. As expected, structures with a greater SASA resulted in dimers with lower stabilities than structures with a smaller SASA. As was done in Figure 2a, the data in Figure 2b were fit to linear trends to be used to predict DS as a function of SASA. Two separate trendlines are shown to distinguish between structures with different ring types.

Interestingly, the size/type of aromatic rings in a molecule affects DS. Structures with five- and six-membered rings produce less stable dimers than structures consisting of only six-membered rings with equivalent SASAs. To examine this observation, we compared the FESs of molecules with equivalent numbers of carbon atoms and rings ( $\mathrm{N}=32,10$ rings $)$, but with differing numbers of fivesix-membered rings: $10 \mathrm{R}$ is composed of ten six-membered rings, M2 is composed of seven, and M1 is composed of six. The results are shown in Figure 3. As anticipated, the general trend is an increase in DS with an increase in the number of six-membered rings. However, the exact trend is hard to quantify as the relationship between DS and ring type is not linear. Future work will address this in more detail. Variation in ring size only causes a small difference in the trends observed in Figure 2b, suggesting that ring composition has much 
less effect on DS than does SASA. However, ring type will still have an effect on DS between compounds with otherwise similar parameters.

Up to this point, all the compounds in our study have been aromatic, but compounds with aliphatic chains are also present in flames as a result of the most common reaction pathways. Indeed, in a high-temperature combustion environment, the most commonly observed types of chains are unsaturated due to the importance of the hydrogen abstraction, acetylene addition (HACA) mechanism in adding a vinyl chain to a ring structure [30]. The stabilization effects due to the presence of aliphatic chains are thoroughly analyzed in another paper [31]. As shown in that work, the presence of any number of vinyl chains on a molecule results in a constant increase in DS. As an example, we compare the FESs of M4 and M5 at $1000 \mathrm{~K}$ (Figure S1). There is a stabilization gain of roughly $0.08 \mathrm{~kJ} / \mathrm{mol} / \mathrm{N}$ in the molecule containing an aliphatic chain. This contribution is relevant only when comparing molecules with small changes in mass.

The previous results show that size is the most relevant factor in homomolecular dimerization stability. Therefore, we restricted our study of heteromolecular dimerization to size dependency. While in general the free energy will depend on a combination of properties of both molecules, the strong dependency on carbon density and the number of carbons suggests that the limiting factor in DS is the size of the smallest molecule. We tested this hypothesis by looking at the problem from two angles. First, we kept the size of the smallest monomer constant and gradually increased the size of the largest monomer. We started with pyrene, whose dimers are unstable at $1000 \mathrm{~K}$ by several factors of the average thermal fluctuation, and worked our way up to ovalene, which shows stable dimers at the same temperature. Second, we kept the size of the largest monomer (ovalene) constant and gradually increased the size of the smallest monomer. The resulting FESs are given in Figure $4 \mathrm{a}$ and Figure $4 \mathrm{~b}$, respectively. The free energy curves show little change when increasing the size of the largest monomer and a vast change when increasing the size of the smallest monomer. Taken together, the results show that stability in hetero-molecular dimerization 
depends mostly on the size of the smaller monomer. A likely explanation for this is that the smaller monomer can only physically interact with a portion of the largest monomer of the same size so intuitively this result is reasonable.

To demonstrate how structural parameters can be used to predict dimer stabilities of new compounds, the fitted trends to the DS as a function of monomer size and SASA at $1000 \mathrm{~K}$ were applied to a test molecule. For this purpose, we selected M2 as a test molecule since it was not part of the data set used for the fitting analysis; M2 is composed of five- and six-membered rings. To test our prediction we reconstructed the free energy of dimerization of the test molecule at $1000 \mathrm{~K}$ following the same methodologies employed above and compared the results to the fitted trends.

The DS of the test case, M2, was well-predicted by the fitted trend for monomer size, with an error of about 4\% (Table S2). This error is considerably less than the average error of the points used to construct the trendline (15\%). The DS was under-predicted for five- and six-membered ring structures, by about $83 \%$ (Table S6). However, the trend developed from five- and six-membered rings is only based on three data points, which span a small range of SASAs. By including molecules containing six-membered rings in the data set, results improve so that DS is only under-predicted by $26 \%$ (Table S7). One explanation for these discrepancies is the limited data set used to develop the trends, an aspect that will be addressed by future work. In sum, although monomer size and SASA appeared to be the most dominant variables governing DS, other factors such as ring type and aliphatic chains cannot be ignored. That is, both mass and SASA are examples of properties that can be leveraged to develop quantitative relationships for dimer stabililty. Moreover, this study represents a framework for quantifying the dimerization behavior of arbitrary PAHs. Further work will be necessary to classify and prioritize additional morphological parameters to build a strong predictive capability that will significantly enhance modeling of particle nucleation. 


\section{Conclusions}

In this paper we have provided a method for linking molecular properties to details about the thermodynamic stability of PAH dimers. We assessed the thermodynamic DS by measuring free energy profiles and found that dimer propensities could be accurately and easily predicted from composition and structural parameters. We found that DS reaches a plateau for large PAHs, is directly correlated to particle morphology, and mainly depends on the size of the smallest monomer in hetero-molecular dimerization. These findings can be used to formulate a physical sticking probability in a combustion environment for arbitrary complex molecules. Further, these free energies have been derived from entirely theoretical constructs, making them adaptable to a range of flame conditions without the need for empirical parameters. This will allow us to model a realistic scenario for particle nucleation, one which includes both chemical and physical growth phenomena. Additionally, our improved model for particle nucleation will allow us to extract atomistic details about the structure of nascent, or newly formed, soot. Future work will seek to improve upon the relationships we obtained by identifying additional factors that influence DS.

\section{Acknowledgements}

This work is supported by the US Department of Energy, Division of Chemi-

cal Sciences, Geosciences, and Biosciences, Contract No. DE-SC0002619. Jason Lai thanks the Natural Science and Engineering Research Council of Canada for his NSERC PGS-D graduate fellowship.

\section{References}

[1] H. Wang, Proceedings of the Combustion Institute 33 (2011) 41-67.

[2] A. D'Anna, Proceedings of the Combustion Institute 32 (2009) 593-613.

[3] M. Frenklach, Physical Chemistry Chemical Physics 4 (2002) 2028-37. 
[4] H. Richter, J. Howard, Progress in Energy and Combustion Science 26 (2000) 565-608.

[5] C. Jäger, H. Mutschke, T. Henning, F. Huisken, European Astronomical Society Publications Series 46 (2011) 293-304.

[6] Z. A. Mansurov, Combustion, Explosion and Shock Waves 41 (2005) 72744.

[7] Z. A. Mansurov, Journal of Engineering Physics and Thermophysics 84 (2011) 125-59.

[8] A. E. Karataş, Ö. L. Gülder, Progress in Energy and Combustion Science 38 (2012) 818-45.

[9] J. Xi, B.-J. Zhong, Chemical Engineering \& Technology 29 (2006) 665-673.

[10] J. Singh, R. I. Patterson, M. Kraft, H. Wang, Combustion and Flame 145 (2006) 117-27.

[11] C. A. Schuetz, M. Frenklach, Proceedings of the Combustion Institute 29 (2002) 2307-14.

[12] J. H. Miller, Symposium (International) on Combustion 23 (1991) 91-8.

[13] H. Sabbah, L. Biennier, S. J. Klippenstein, I. R. Sims, B. R. Rowe, The Journal of Physical Chemistry Letters 1 (2010) 2962-7.

[14] S.-H. Chung, A. Violi, Proceedings of the Combustion Institute 33 (2011) 693-700.

[15] P. Elvati, A. Violi, Proceedings of the Combustion Institute 34 (2013) 183743.

[16] T. S. Totton, A. J. Misquitta, M. Kraft, Physical Chemistry Chemical Physics 14 (2012) 4081-94.

[17] M. Sander, R. I. Patterson, A. Braumann, A. Raj, M. Kraft, Proceedings of the Combustion Institute 33 (2011) 675-83. 
[18] D. Chen, Z. Zainuddin, E. Yapp, J. Akroyd, S. Mosbach, M. Kraft, Proceedings of the Combustion Institute 34 (2013) 1827-35.

[19] A. Raj, M. Sander, V. Janardhanan, M. Kraft, Combustion and Flame 157 (2010) 523-34.

[20] A. D'Anna, M. Sirignano, J. Kent, Combustion and Flame 157 (2010) 2106-15.

[21] A. Barducci, G. Bussi, M. Parrinello, Physical Review Letters 100 (2008) 20603.

[22] J. D. Herdman, J. H. Miller, The Journal of Physical Chemistry A 112 (2008) 6249-56.

[23] W. L. Jorgensen, D. S. Maxwell, J. Tirado-Rives, Journal of the American Chemical Society 118 (1996) 11225-36.

[24] S. Plimpton, Journal of Computational Physics 117 (1995) 1-19.

[25] M. Bonomi, D. Branduardi, G. Bussi, C. Camilloni, D. Provasi, P. Raiteri, D. Donadio, F. Marinelli, F. Pietrucci, R. a. Broglia, M. Parrinello, Computer Physics Communications 180 (2009) 1961-72. doi:10.1016/j.cpc.2009.05.011.

[26] W. Humphrey, A. Dalke, K. Schulten, Journal of molecular graphics 14 (1996) 33-8.

[27] S. E. Stein, A. Fahr, The Journal of Physical Chemistry 89 (1985) 3714-25.

[28] J. Y. W. Lai, P. Elvati, A. Violi, Physical chemistry chemical physics : PCCP 16 (2014) 7969-79.

[29] A. Violi, A. Venkatnathan, The Journal of Chemical Physics 125 (2006) 054302-.

[30] M. Frenklach, H. Wang, Symposium (International) on Combustion 23 (1991) 1559-66. 
[31] J. S. Lowe, P. Elvati, A. Violi, In preparation (2014). 
Tables

Table 1: Parameters for molecular dynamics simulations.

\begin{tabular}{cc} 
Parameter & Value \\
\hline Thermostat & Langevin $(\tau=5 \mathrm{fs})$ \\
Time step & $1 \mathrm{fs}$ \\
Non-bonded cutoff & $1.4 \mathrm{~nm}$ \\
Accuracy for particle-particle particle-mesh solver & $1.0 \mathrm{e}-4$ \\
Gaussian height & $2.092 \mathrm{~kJ} / \mathrm{mol}$ \\
Gaussian sigma & $0.02 \mathrm{~nm}$ \\
Bias factor & $3-42$ \\
Time between Gaussian deposition & $200 \mathrm{fs}$
\end{tabular}




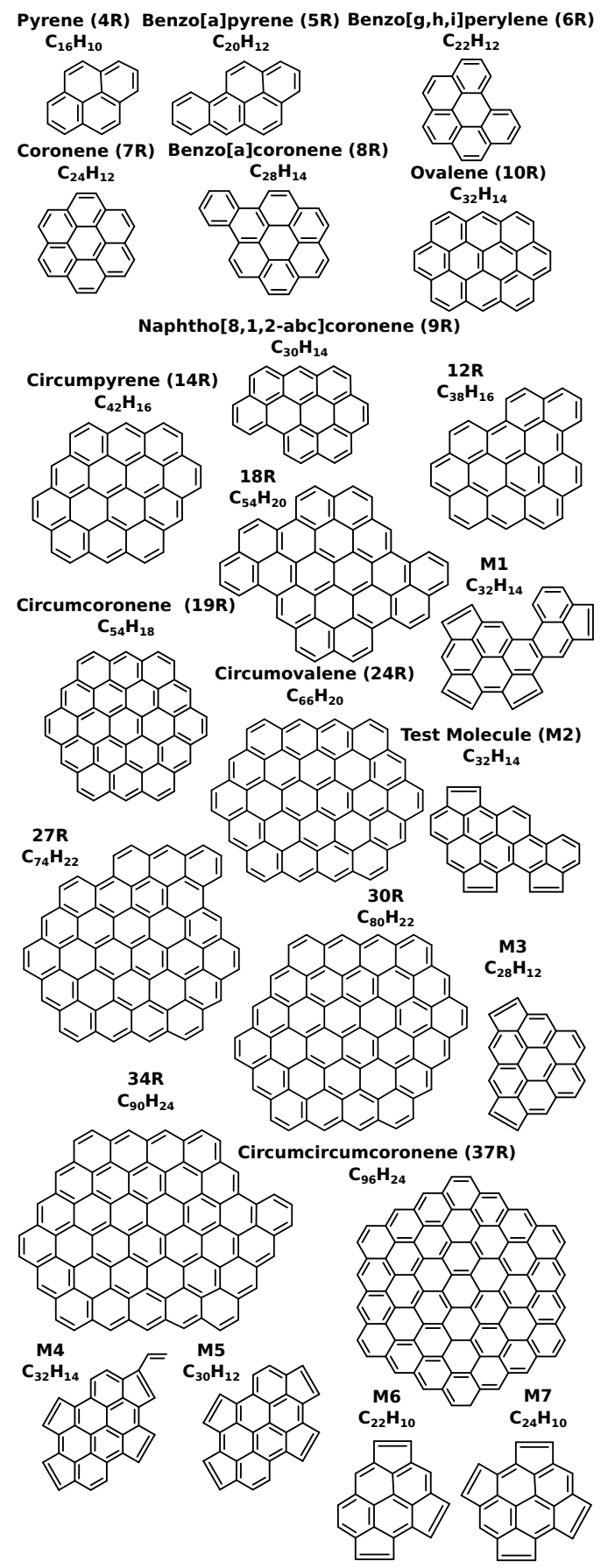

Figure 1: Pool of molecules involved in study. 


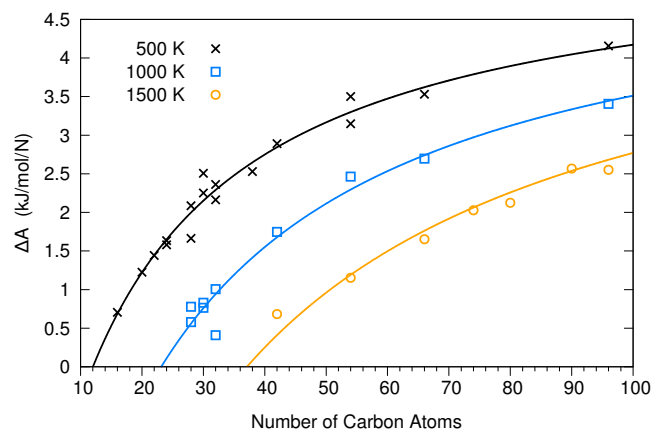

(a)

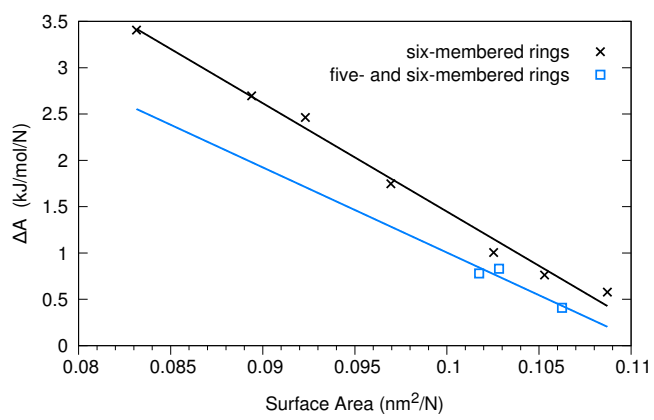

(b)

Figure 2: Free energy of dimerization for aromatic, homo-molecular species as a function of: (a) carbon number at different temperatures and (b) SASA per carbon at $1000 \mathrm{~K}$. The curves shown in each plot are fitted to the data using the following functional forms: (a) $F E / N=a_{0}-a_{1}\left(N-a_{2}\right)^{-1}$ and (b) a simple linear trend. The error is about $0.22 \mathrm{~kJ} / \mathrm{mol} / \mathrm{N}$ at $500 \mathrm{~K}, 0.11 \mathrm{~kJ} / \mathrm{mol} / \mathrm{N}$ at $1000 \mathrm{~K}$, and $0.22 \mathrm{~kJ} / \mathrm{mol} / \mathrm{N}$ at $1500 \mathrm{~K}$. Deviation of the data from the fitted trendlines is summarized in tables S1-S3, S6, and S8 in the Supplementary Material. 


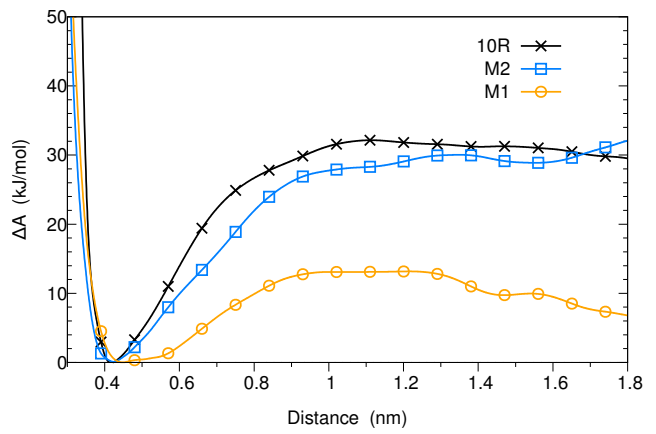

Figure 3: Free energy of dimerization surfaces for homo-molecular pairs as a function of the center of mass distance between monomers. Each molecule is composed of ten total rings, but they differ by the number of six-membered rings: 10R has ten, M2 has seven, and M1 has six. 


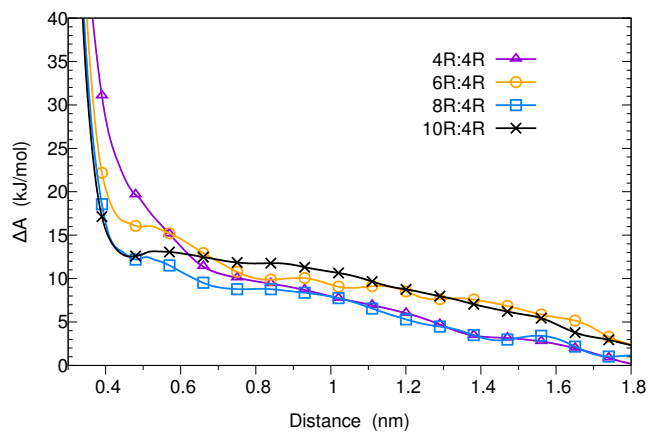

(a)

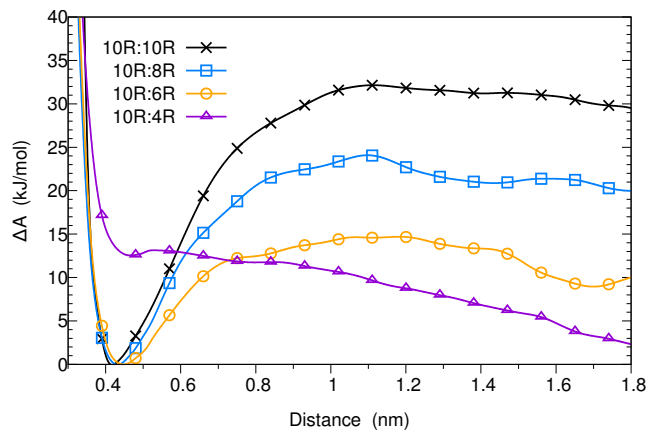

(b)

Figure 4: Free energy surfaces of dimerization for hetero-molecular pairs over the center of mass distance collective variable as a function of: (a) the largest monomer and (b) the smallest monomer. 


\section{Figure Captions}

\section{List of Figures}

1 Pool of molecules involved in study. . . . . . . . . . . . . 16

2 Free energy of dimerization for aromatic, homo-molecular species as a function of: (a) carbon number at different temperatures and (b) SASA per carbon at $1000 \mathrm{~K}$. The curves shown in each plot are fitted to the data using the following functional forms: (a) $F E / N=a_{0}-a_{1}\left(N-a_{2}\right)^{-1}$ and (b) a simple linear trend. The error is about $0.22 \mathrm{~kJ} / \mathrm{mol} / \mathrm{N}$ at $500 \mathrm{~K}, 0.11 \mathrm{~kJ} / \mathrm{mol} / \mathrm{N}$ at 1000 $\mathrm{K}$, and $0.22 \mathrm{~kJ} / \mathrm{mol} / \mathrm{N}$ at $1500 \mathrm{~K}$. Deviation of the data from the fitted trendlines is summarized in tables S1-S3, S6, and S8 in the Supplementary Material. . . . . . . . . . . . . . . . 17

3 Free energy of dimerization surfaces for homo-molecular pairs as a function of the center of mass distance between monomers. Each molecule is composed of ten total rings, but they differ by the number of six-membered rings: 10R has ten, M2 has seven, and M1 has six. . . . . . . . . . . . . . . . . . . . 18

4 Free energy surfaces of dimerization for hetero-molecular pairs over the center of mass distance collective variable as a function of: (a) the largest monomer and (b) the smallest monomer. . . . 19 